\title{
Types of deviation and review criteria in pretreatment central quality control of tumor bed boost in medulloblastoma-an analysis of the German Radiotherapy Quality Control Panel in the SIOP PNET5 MB trial
}

\author{
Stefan Dietzsch ${ }^{1,2}$ (D) Annett Braesigk ${ }^{1}$. Clemens Seidel ${ }^{1}$ Julia Remmele ${ }^{1}$. Ralf Kitzing ${ }^{1} \cdot$ Tina Schlender $^{1}$. \\ Martin Mynarek ${ }^{3}$ - Dirk Geismar ${ }^{2}$ Karolina Jablonska ${ }^{4}$ Rudolf Schwarz ${ }^{5}$ Montserrat Pazos ${ }^{6}$. \\ Damien C. Weber ${ }^{7}$. Silke Frick ${ }^{8}$. Kristin Gurtner ${ }^{9}$ Christiane Matuschek ${ }^{10}$ • Semi Ben Harrabi ${ }^{11}$. \\ Albrecht Glück ${ }^{12}$ • Victor Lewitzki ${ }^{13}$ - Karin Dieckmann ${ }^{14}$ - Martin Benesch ${ }^{15}$ • Nicolas U. Gerber ${ }^{16}$. \\ Denise Obrecht $^{3} \cdot$ Stefan Rutkowski ${ }^{3}$ - Beate Timmermann ${ }^{2} \cdot$ Rolf-Dieter Kortmann ${ }^{1}$
}

Received: 8 February 2021 / Accepted: 2 July 2021 / Published online: 5 August 2021

(c) The Author(s) 2021

\begin{abstract}
Purpose In Germany, Austria, and Switzerland, pretreatment radiotherapy quality control (RT-QC) for tumor bed boost (TB) in non-metastatic medulloblastoma (MB) was not mandatory but was recommended for patients enrolled in the SIOP PNET5 MB trial between 2014 and 2018. This individual case review (ICR) analysis aimed to evaluate types of deviations in the initial plan proposals and develop uniform review criteria for TB boost.

Patients and methods A total of 78 patients were registered in this trial, of whom a subgroup of 65 patients were available for evaluation of the TB treatment plans. Dose uniformity was evaluated according to the definitions of the protocol. Additional RT-QC criteria for standardized review of target contours were elaborated and data evaluated accordingly.
\end{abstract}

Data sharing statement Research data are stored in an institutional repository and will be shared upon request to the corresponding author.

B. Timmermann and R-D. Kortmann share last authorship.

Dr. med. Stefan Dietzsch, MD

stefan.dietzsch@medizin.uni-leipzig.de

1 Department of Radiation Oncology, University of Leipzig Medical Center, Stephanstr. 9a, 04103 Leipzig, Germany

2 Clinic for Particle Therapy, West German Proton Therapy Centre, University of Essen, Essen, Germany

3 Departement of Pediatric Hematology and Oncology, University Medical Center Hamburg-Eppendorf, Hamburg, Germany

4 Faculty of Medicine, Department of Radiation Oncology, University of Cologne, Cologne, Germany

5 Department of Radiation Oncology, University Medical Center Hamburg-Eppendorf, Hamburg, Germany

6 Department of Radiotherapy and Radiation Oncology, Ludwig Maximilian University Munich, Munich, Germany

7 Center for Protontherapy, Paul Scherrer Institute, Villigen, Switzerland
8 Department of Radiotherapy and Radiation Oncology, Hospital Bremen Mitte, Bremen, Germany

9 Department of Radiotherapy and Radiation Oncology, Faculty of Medicine and University Hospital, Carl Gustav Carus, Technical University Dresden, Dresden, Germany

10 Department of Radiation Oncology, Medical Faculty Heinrich Heine University Duesseldorf, Duesseldorf, Germany

11 Department of Radiation Oncology and Radiotherapy, Heidelberg University Hospital, Heidelberg, Germany

12 Radiation Oncology, Munich-Schwabing Municipal Hospital, Munich, Germany

13 Department of Radiotherapy, University of Wuerzburg, Wuerzburg, Germany

14 Department of Radiotherapy, Medical University of Vienna, Vienna, Austria

15 Division of Pediatric Hematology/Oncology, Department of Pediatrics and Adolescent Medicine, Medical University of Graz, Graz, Austria

16 Department of Oncology, University Children's Hospital, Zurich, Switzerland 
Results Of 65 initial TB plan proposals, 27 (41.5\%) revealed deviations of target volume delineation. Deviations according to the dose uniformity criteria were present in $14(21.5 \%)$ TB plans. In $25(38.5 \%)$ cases a modification of the RT plan was recommended. Rejection of the TB plans was rather related to unacceptable target volume delineation than to insufficient dose uniformity.

Conclusion In this analysis of pretreatment RT-QC, protocol deviations were present in a high proportion of initial TB plan proposals. These findings emphasize the importance of pretreatment RT-QC in clinical trials for MB. Based on these data, a proposal for RT-QC criteria for tumor bed boost in non-metastatic MB was developed.

Keywords Brain tumor · Pediatric · Focal radiotherapy - Quality assurance · Individual case review

\section{Introduction}

Postoperative craniospinal irradiation (CSI) with boost delivery is a cornerstone of treatment of medulloblastomas (MB) [1]. In standard-risk patients, the boost volume has changed from the posterior fossa $(\mathrm{PF})$ to the tumor bed (TB) [2]. For PF boost, retrospective reports showed that inadequate treatment volumes were applied in a substantial proportion of patients [3-5]. A retrospective analysis of the French Medulloblastome-Société Française d'Oncologie Pédiatrique 1998 (M-SFOP 98) trial revealed adequate target volume and dose distribution of tumor bed boost [6]. Experiences with focal radiotherapy in other brain tumors using similar target volume concepts, however, showed significant rates of radiotherapy (RT) protocol deviations [7-10].

The International Society of Pediatric Oncology Peripheral Primitive Neuroectodermal Tumor $5 \mathrm{MB}$ trial (SIOP PNET5 MB, ClinicalTrials.gov identifier: NCT02066220) included a TB boost with a reduced clinical target volume (CTV) margin of $1.0 \mathrm{~cm}$ compared to previously published trials [6, 11, 12]. Protocol-compliant application of the TB boost was required to draw conclusions on the CTV margin out of the relapse patterns inside the posterior fossa. This report presents first data of pretreatment radiotherapy quality control (RT-QC) of TB boost in patients enrolled to the trial in Germany, Switzerland, and Austria between 2014 and 2018. The analysis details the types of deviations observed in the initial plan proposals submitted by local departments of radiation oncology and describes uniform review criteria for future MB studies. The pattern of relapse will be a subject of the final analysis of the protocol.

\section{Patients and methods}

In the SIOP PNET5 MB trial, pretreatment central RT-QC of CSI plans was mandatory. RT-QC was organized on a national basis. Data on patients enrolled in Germany, Switzerland, and Austria, including details on treatment schedule, treatment techniques, workflow of RT-QC, results of RTQC of CSI plans, and the German radiotherapy control panel, have been published elsewhere [13]. In the first years of trial recruitment, transfer and central review of Digital Imaging and Communications in Medicine (DICOM)-RT data with Magnetic Resonance Imaging (MRI) fusion could not be ensured in all participating countries. Therefore, pretreatment RT-QC of TB boost was optional in the first protocol versions (version 10, February 21, 2013; and version 11, November 17, 2014). However, pretreatment RTQC was recommended by the German radiotherapy control panel. According to the protocol, the national radiotherapy QC panels were responsible for determining the criteria by which TB boost treatment plans were evaluated. For dose uniformity, minor and major deviations were used prospectively, which were defined in consensus of the national RT coordinators according to International Commission on Radiation Units and Measurements (ICRU) 50/62 and which were also used for RT-QC of 3D conformal CSI plans from the beginning of the central review and incorporated into the protocol version 12 (June 29, 2017) [14]. The criteria are described in Table 1. This protocol version was initiated in Germany in November 2018 and did not include definitions of target volume deviations for TB boost. For target delineation, RT-QC criteria were adapted from the SIOP Ependymoma II protocol (ClinicalTrials.gov identifier: NCT02265770) for evaluation (Table 1). Furthermore, deviations were rated for clinical relevance and scored as "acceptable" or "unacceptable" as described elsewhere [13].

Details of the treatment recommendations of the protocol were presented and discussed with the local radiation oncologists at meetings of the Arbeitsgemeinschaft pädiatrische Radioonkologie (APRO; Working Group Pediatric Radiation Oncology). A benchmark case was not performed. Protocol deviations were communicated to the local radiation oncologists via telephone or email including illustrating screenshots.

Central plan analyses were performed using the treatment planning systems (TPS) of the reference center (RayStation, Raysearch Laboratories, Stockholm, Sweden). The original dose file of the local radiotherapy was imported and evaluated. A re-calculation was not done, only a re-sampling of dose-volume parameters was performed in case of modified volumes of interest. Use of the original 
Table 1 Protocol definitions of RT parameters for quality control and additional definitions of minor and major deviations in target delineation used by the reference center

\begin{tabular}{|c|c|c|c|}
\hline & Per protocol & $\begin{array}{l}\text { Minor devi- } \\
\text { ation }\end{array}$ & Major deviation \\
\hline GTV & $\begin{array}{l}\text { Resection } \\
\text { cavity and/or } \\
\text { residual tumor }\end{array}$ & Not defined & Not defined \\
\hline CTV & $\begin{array}{l}\mathrm{GTV}+1 \mathrm{~cm} \\
\text { except bone } \\
\text { and tentorium }\end{array}$ & Not defined & Not defined \\
\hline PTV & $\begin{array}{l}\mathrm{CTV}+0.3 \text { to } \\
0.5 \mathrm{~cm}\end{array}$ & Not defined & Not defined \\
\hline \multicolumn{4}{|c|}{ Dose uniformity } \\
\hline V95\% & $\geq 95 \%$ & $\begin{array}{l}\geq 90 \text { to } \\
<95 \%\end{array}$ & $<90 \%$ \\
\hline V107\% & $\leq 5 \%$ & $\begin{array}{l}>5 \% \text { to } \\
<10 \%\end{array}$ & $\geq 10 \%$ \\
\hline \multicolumn{4}{|c|}{$\begin{array}{l}\text { Additional definitions of target delineation minor and major devia- } \\
\text { tions }\end{array}$} \\
\hline CTV & $1.0 \mathrm{~cm}$ & $\begin{array}{l}<1.0 \mathrm{~cm} \\
\text { PTV mar- } \\
\text { gin }\end{array}$ & Not defined \\
\hline GTV & - & $\begin{array}{l}\text { Encompass } \\
\text { normal } \\
\text { brain tissue }\end{array}$ & $\begin{array}{l}\text { Not encompass MRI } \\
\text { visible resection cav- } \\
\text { ity/residual tumor }\end{array}$ \\
\hline
\end{tabular}

GTV gross tumor volume, $C T V$ clinical target volume, $P T V$ planning target volume, V95 volume of PTV receiving $\geq 95 \%$ of the prescribed dose, V107 volume of PTV receiving $\geq 107 \%$ of the prescribed dose
MRI-CT co-registration matrix was established at the end of the observed period. Therefore, new image registrations with the cranial MRIs were performed in almost all cases, taking into account the uncertainties of co-registration differences between TPS of the local radiotherapy unit and the system of the reference center.

Associations between variables were examined using Fisher exact and $\chi^{2}$ tests. All statistical analyses were performed using the Statistical Package for Social Sciences (IBM SPSS statistics), version 24 (IBM, Armonk, NY, USA).

\section{Results}

Between September 2014 and December 2018, 78 patients (Germany, $n=70$; Switzerland, $n=6$, and Austria, $n=2$ ) were enrolled in the SIOP-PNET5-MB trial. DICOM-RT data were unavailable in 13 (19\%) patients; thus, 65 boost $(81 \%)$ plans were analyzed. The majority $(41 / 65 ; 63.1 \%)$ of the patients were treated according to the SIOP PNET5 SR arm (CSI 23.4Gy + TB boost 30.6 Gy) and 24/65 patients (36.9\%) according to the arm for patients with a low-risk biological profile (SIOP PNET5 LR arm, CSI 18.0 Gy + TB boost $36.0 \mathrm{~Gy})$. Pre- and post-surgery MRIs were available for review in $46(70.8 \%)$ patients, whereas 7 patients (10.8\%) had only post-surgery MRI and 1 patient (1.5\%) only pre-surgery MRI. In 11 patients (16.9\%) MRI was not available for plan review (Fig. 1).

Target volume deviations occurred in $41.5 \%$ of cases (Supplementary Table 1). The most common reason for target volume deviation was incorrect clinical target volume (CTV) and/or planning target volume (PTV) margin
Fig. 1 Consort diagram of the present analysis. SIOP-PNET5$M B$ The International Society of Pediatric Oncology Peripheral Primitive Neuroectodermal Tumor 5 Medulloblastoma trial, DICOM Digital Imaging and Communications in Medicine, $R T$ radiotherapy, $Q C$ quality control, MRI magnetic resonance imaging, $S R$ standard risk, $L R$ low risk

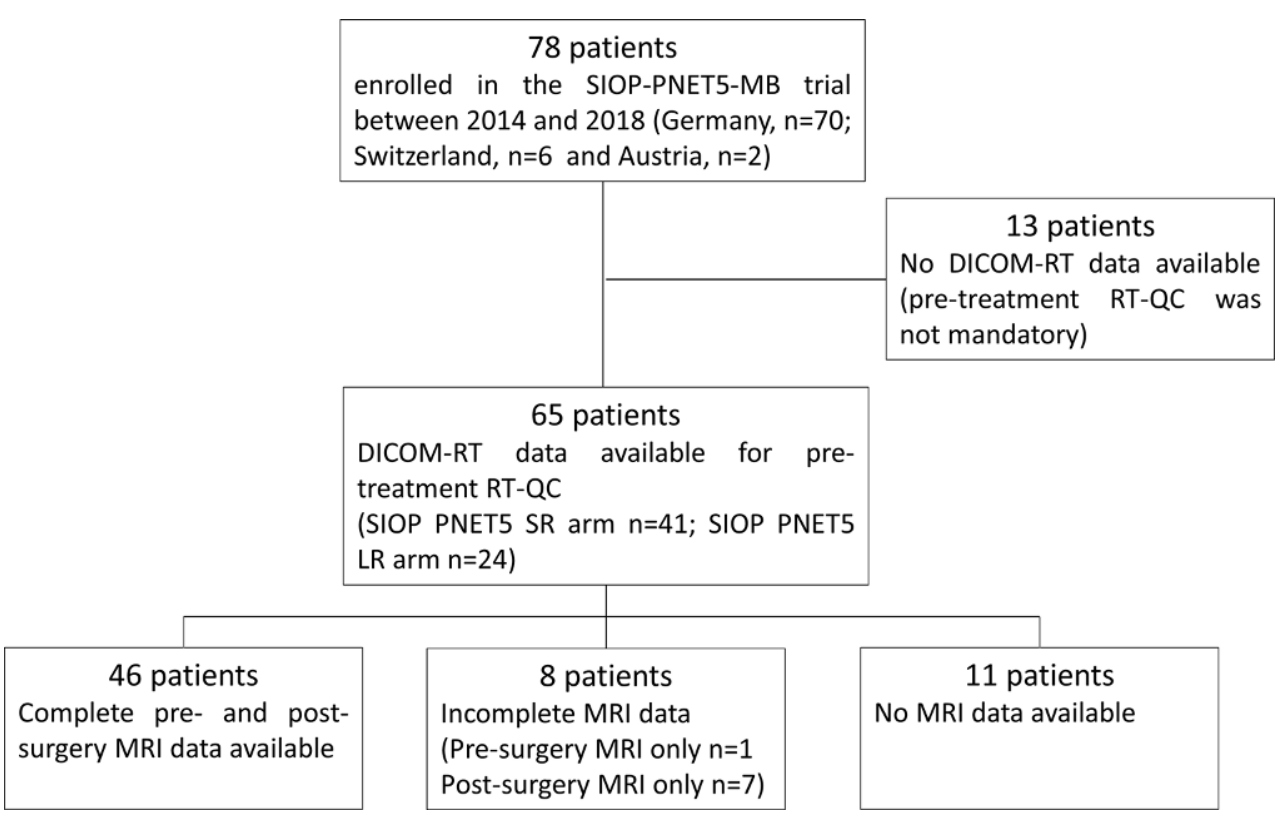



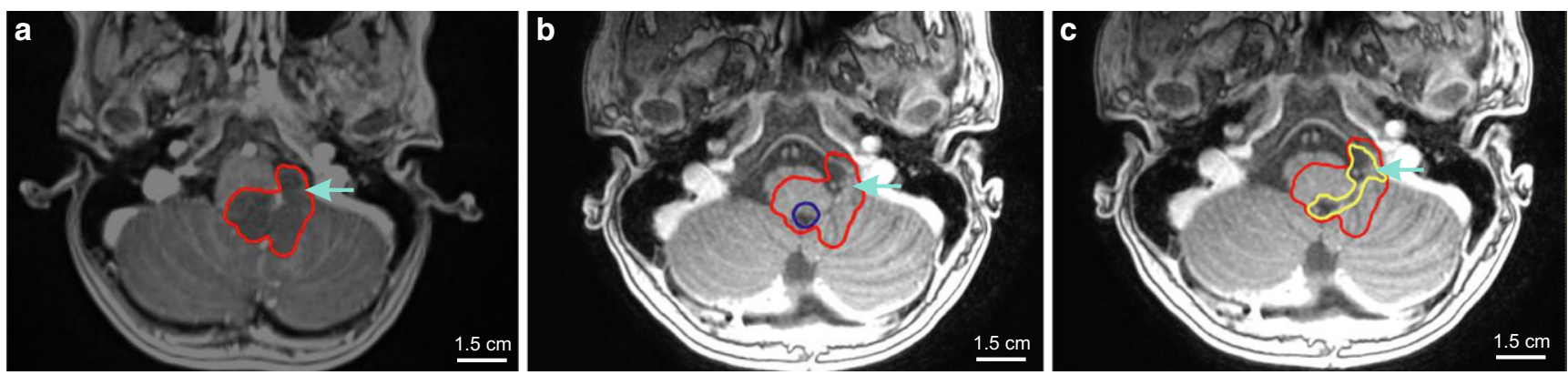

Fig. 2 Example of a major/unacceptable deviation; a shows the pre-surgery MRI with the initial tumor volume (red); b shows the post-surgery MRI with the $\mathrm{GTV}_{\text {tumorbed }}$ (blue) of the local radiation oncologist, which does not encompass the MRI visible resection cavity/areas with initial tumor contact (initial tumor volume, red); $\mathbf{c}$ shows the reference $\mathrm{GTV}_{\text {tumorbed }}$ (yellow) and the initial tumor volume (red). The green arrow marks the region of deviation. MRI magnetic resonance imaging, GTV gross tumor volume

(26.2\%; Supplementary Figure 1). Delineation of resection cavity (gross tumor volume, $\mathrm{GTV}_{\text {tumorbed }}$ ) was incorrect in $20.0 \%$ of plans. Predominantly, $\mathrm{GTV}_{\text {tumorbed }}$ was too large and encompassed normal brain tissue. In 4 patients, $\mathrm{GTV}_{\text {tumorbed }}$ did not encompass the complete resection cavity (Fig. 2). In 14 patients without complete MRI data, no deviation of $\mathrm{GTV}_{\text {tumorbed }}$ was detected when reviewing the planning CT. However, a final judgement on correctness of the $\mathrm{GTV}_{\text {tumorbed }}$ was not possible in these patients.

In 5 of $53(9.4 \%)$ cases with available post-surgery MRI, a striking discrepancy between the resection cavity on MRI, performed within $48 \mathrm{~h}$ after surgery, and the planning CT was found (Supplementary Figure 2). The interval between post-surgery MRI and planning CT was median 29 (range 18 to 35 ) days in these patients.

Dose uniformity deviations were found in $23.1 \%$ of plans and were predominantly minor $(18.5 \%)$. The median V95 (volume of PTV receiving $\geq 95 \%$ of the prescribed dose) was $98.5 \pm 3.3 \%$. A V95<95\% was observed in $14(21.5 \%)$ of plans. In one plan $(1.5 \%)$ the V107 (volume of PTV receiving $\geq 107 \%$ of the prescribed dose) was more than
$5 \%$. RT technique had no impact on the frequency of dose uniformity deviations (Supplementary Table 2; Fisher exact test, $p=0.509$ ).

Overall, 34 plans (52.3\%) did not reveal any deviation (Supplementary Table 3). In 9.2\% of plans, deviations were considered acceptable and in 25 (38.5\%) plans, modifications were recommended. In the cohort of plans with deviations $(n=31)$, no significant correlation between the defined minor and major deviations and the acceptance of plans was seen (unacceptable plans 71.4 vs. $83.3 \%$; chi $^{2} p=0.483$, Table 2). Moreover, there was no difference in the rate of unacceptable plans between patients with or without complete MRI data (37.0 vs. $42.1 \%$; $\left.\mathrm{chi}^{2} p=0.875\right)$. However, 10 of the 14 patients with incomplete MRI data and no deviation of $\mathrm{GTV}_{\text {tumorbed }}$ detected in the planning CT were finally scored as acceptable with the remark of incomplete review.

There was no difference in the need for plan modifications between low-recruiting ( $\leq 4$ patients) and high-recruiting $\left(\geq 5\right.$ patients) radiotherapy units $\left(42.3 \%\right.$ vs. $35.9 \%$; chi $^{2}$ $p=0.461)$. Fig. 3 shows the impact of institutional experiences in treating SIOP PNET5 MB trial patients on the

Table 2 Relationship between types of deviation (target volume and/or dose uniformity) and overall result of quality control (acceptable/ unacceptable)

\begin{tabular}{|c|c|c|c|c|}
\hline & $\begin{array}{l}\text { Per proto- } \\
\text { col }\end{array}$ & Acceptable deviation & Unacceptable deviation & Total \\
\hline Correct target and dose & 34 & 0 & 0 & $\begin{array}{l}34 \\
(52.3 \%)\end{array}$ \\
\hline $\begin{array}{l}\text { Correct target but deviation } \\
\text { dose uniformity }\end{array}$ & 0 & $\begin{array}{l}3 \\
\text { dose minor } n=2 \\
\text { dose major } 0=1\end{array}$ & $\begin{array}{l}1 \\
\text { dose major } n=1\end{array}$ & $4(6.2 \%)$ \\
\hline $\begin{array}{l}\text { Deviation target but correct } \\
\text { dose uniformity }\end{array}$ & 0 & $\begin{array}{l}2 \\
\text { target minor } n=1 \\
\text { target major } n=1\end{array}$ & $\begin{array}{l}14 \\
\text { target minor } n=12 \\
\text { target major } n=2\end{array}$ & $\begin{array}{l}16 \\
(24.6 \%)\end{array}$ \\
\hline $\begin{array}{l}\text { Deviation target and dose uni- } \\
\text { formity }\end{array}$ & 0 & $\begin{array}{l}1 \\
\text { dose minor } n=1 \\
\text { target minor } n=1\end{array}$ & $\begin{array}{l}10 \\
\text { dose minor } n=9 \\
\text { dose major } n=1 \text { target minor } n=9 \\
\text { target major } n=1\end{array}$ & $\begin{array}{l}11 \\
(16.9 \%)\end{array}$ \\
\hline Total & $34(52.3 \%)$ & $6(9.2 \%)$ & $25(38.5 \%)$ & $\begin{array}{l}65 \\
(100 \%)\end{array}$ \\
\hline
\end{tabular}


Fig. 3 Influence of experience within the protocol on quality control result. $n$ depicts number of patients who were evaluated with respect to the patients in radiotherapy unit (e.g., 23 patients were evaluated as the first patient in an unit)

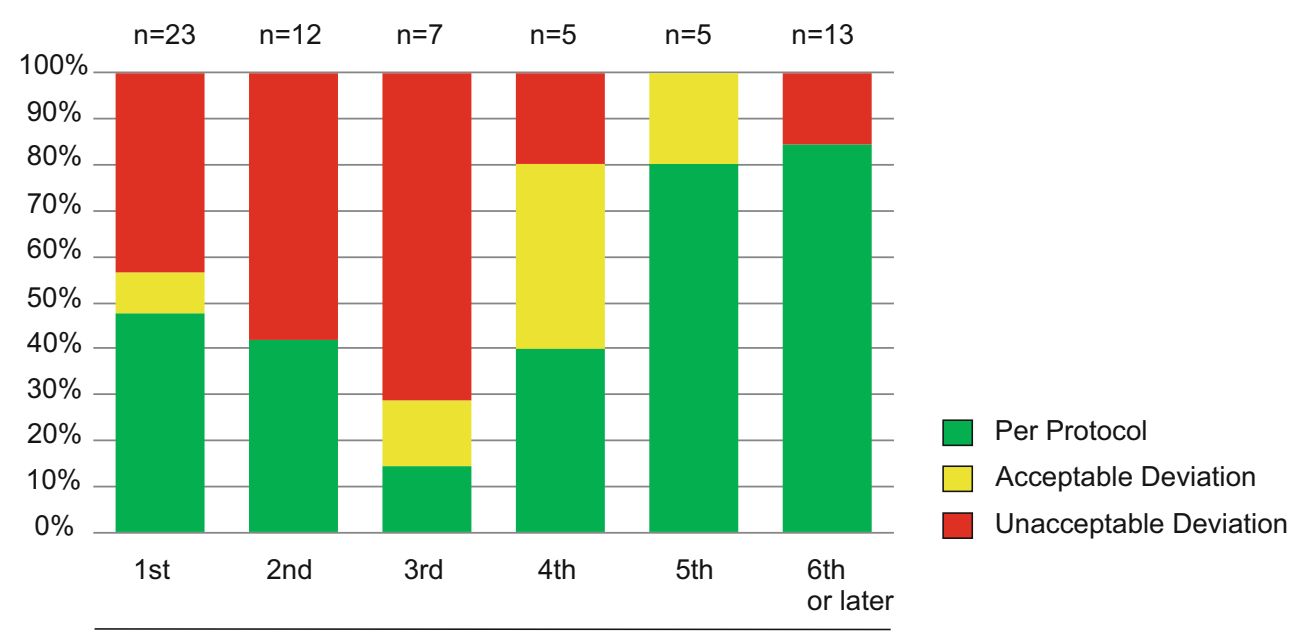

Patient in RT Unit rate of unacceptable RT plans. Most of the unacceptable boost plans occurred in the first to third patient of each radiotherapy unit $(22 / 25 ; 88 \%)$. The decline of the ratio was significant (first to third patient in unit $52.4 \%$ vs. fourth or later patient in unit $13.0 \% ; p=0.008)$. However, 3 cases of unacceptable plans were also observed in the second group (fouth or later patient in unit).

Table 2 summarizes the type of observed deviation and the need for plan modification. Only 1 plan with dose uniformity deviation alone required plan modification. In 14 of $25(56 \%)$ cases with unacceptable deviations, only target volume deviations were present. Of these patients $2 / 14$ (14.3\%) had major deviations (GTV did not encompass the resection cavity), 1/14 (7.1\%) minor deviation (GTV encompassed too much normal brain tissue), 10/14 (71.4\%) minor deviations (incorrect CTV/PTV margin), and 1/14 (7.1\%) minor deviation (both, i.e., GTV encompassed too much normal brain tissue and incorrect CTV/PTV margin). In $10 / 25$ patients (40\%), unacceptable plans showed both target volume and dose uniformity deviations.

\section{Discussion}

The present study represents a large cohort of MB patients undergoing a pretreatment, fully digital, individual case review QC procedure in TB boost for MB.

\section{Observed deviations}

We observed RT plan deviations in approximately $50 \%$ of cases and recommended plan modification in $38.5 \%$ of plans. To date, no comparable data on pretreatment RT-QC of 3D conformal TB boost in MB is available. Previous reports examined deviations of simulation-based PF boost and observed frequent inaccuracies. However, most of the deviations were considered minor or seemed to have no significant impact on event-free or overall survival $[3,5,15$, 16]. In contrast, RT-QC analysis in the SIOP United Kingdom Children's Cancer Study Group (UKCCSG) PNET3 trial revealed a significantly higher $\mathrm{PF}$ recurrence rate in patients treated with PF-targeting deviation ( $34.4 \%$ vs. $16.3 \%$; $p=0.043)$. However, no significant impact on overall survival $(p=0.4034)$ was observed [4].

Retrospective quality control of $\mathrm{TB}$ boosts in the FRENCH M-SFOP 98 trial could not show any inadequacies of target definition and dose distribution. However, details and definitions of RT-QC criteria were not provided within that report [6].

The planning procedure of tumor bed boost in MB is comparable to local irradiation of other brain tumor entities. The target volumes consist of the tumor bed and/or residual tumor with an isotropic safety margin for subclinical disease of about 0.5 to $1.5 \mathrm{~cm}$ (CTV margin), which has to be adapted to anatomical borders. The CTV margin depends on the infiltrating potential of the tumor or special character of the tumor, e.g., growing of meningiomas along the meninges. Another safety margin is added for the setup error (PTV margin). In low-grade gliomas, a retrospective RT-QC analysis resulted in overall grades of minor and major deviation of $37 \%$ and $32 \%$, respectively [7]. In malignant/atypical meningiomas, Coskun et al. observed major and minor deviations in $22 \%$ and $10 \%$ of reviewed plans, respectively [8]. The RT-QC of the CATNON intergroup trial included a retrospective individual case review for each first patient randomized per institution: $35.5 \%$ of the cases were evaluated as per protocol, $17.7 \%$ as acceptable variation, and $46.8 \%$ as unacceptable [10].

In our study, we also observed high rates of target volume deviations $(41.5 \%)$. The requirement for boost plan modification was rather related to target deviations than to dose uniformity issues. High rates of target volume vari- 
ations $(71 \%)$ were also observed in the CATNON cohort, resulting in unacceptable variations in $27 \%$ of the cases [10]. Retrospective RT-QC of the EORTC 22033-26033 trial also revealed high rates of incorrect volume delineation (63\% minor, 23\% major deviations) [7]. A high inter-clinician variation in contouring of $\mathrm{TB}$ in $\mathrm{MB}$ was also shown in a planning exercise prior to the SIOP PNET4 trial [17]. These findings point out that target delineation is the most critical part of treatment planning, emphasizing the role of central review of target volumes. Moreover, a training program for all participating investigators (e.g., a benchmark case) with a final certificate would be desirable for further investigations.

In contrast to other studies, in our cohort dose uniformity deviations were less frequent and not necessarily combined with unacceptable plans. This might be explained by the relatively low total dose of $54 \mathrm{~Gy}$ in the SIOP PNET5 study as compared to the EORTC 22042-26042 and CATNON trials with $60 \mathrm{~Gy}$ or more applied nearby critical organs, such as the brainstem or optic pathway, frequently leading to dose reductions $[8,10]$.

\section{Role of MRI co-registration}

Incorrect delineation of $\mathrm{GTV}_{\text {tumorbed }}$ was observed in $20 \%$ of the plans and was comparable to other reports [7, 10]. Coregistration with initial and post-surgery imaging improves the accuracy of target delineation and is now standard for contouring of the resection cavity [18, 19]. Central review was based on CT/MRI co-registration of pre- and postsurgery MRI in $70.8 \%$ of the patients; $12.3 \%$ of patients had incomplete MRI data (only pre- or post-surgery MRI available) and in $16.9 \%$ of patients, no MRI was available. Availability of MRI co-registration was higher than in the cohorts of the EORTC 22042-26042 (36.8\% no MRI data) or CATNON trials (proper co-registration in $42.9 \%$ of patients) [7, 10]. No difference in correlation of unacceptable plans with or without complete MRI data could be revealed. This may partly be explained by the high rate of incorrect CTV/PTV margins (26.2\%) leading to unacceptable plans evaluable without any MRI. On the other hand, in 2 cases, refusal of plans was based on discrepancies between the initial tumor volume and the resection cavity as delineated by the local radiation oncologist without analyzing the MRI co-registration during review. In contrast, the resection cavity was considered correct when reviewing only the planning CT in 14 patients. In these cases, an uncertainty of review remained and was commented in the reviewing report. In the majority of cases, the original coregistration matrix was not available and the reviewer performed a new co-registration. This procedure has to take into account the possibility of co-registration differences. However, these differences seem to be limited in the cra- nial region. A multi-institutional benchmark test for cranial CT/MR image registration revealed an uncertainty of approximately $2 \mathrm{~mm}$ [20]. In 5 patients, we detected a discrepancy between the resection cavity visible in the postsurgery MRI, performed within $48 \mathrm{~h}$ after surgery, and the planning CT. Geometrical changes in the resection cavity before RT have also been reported in other brain tumor entities, e.g., gliomas or brain metastases [21, 22]. This issue needs to be further examined in order to understand whether an additional MRI would be mandatory at the time of RT planning or even during CSI before planning and starting the TB boost.

\section{New RT-QC criteria}

No definitions for target volume deviations of the TB boost were available within the SIOP PNET5 MB protocol. In principle, target delineation of boost volume is comparable to local radiotherapy of other brain tumor entities. However, no consensus on target delineation deviations exists. Definitions differ between available reports (Supplementary Table 4) [7, 8, 10]. We defined incomplete coverage of the resection cavity/residual tumor by the GTV as major deviation. An insufficient margin or inclusion of uninvolved normal brain tissue into the GTV was considered a minor deviation (Table 1). However, no correlation between the defined major deviations and unacceptance of plans on one hand and between minor deviations and acceptance of plans on the other hand was seen. We observed a high number of incorrect CTV margins defined as minor deviation, often leading to the recommendation for plan modification due to an expected increase in the risk of relapse. In 9 cases, the resection cavity was delineated too large and unnecessarily encompassed normal brain tissue without an anticipated increased risk of relapse. Nevertheless, plans were considered unacceptable when a larger volume of uninvolved normal tissue was included due to the potentially higher risk of toxicity. Moreover, creation of the PTV is a multistep approach (GTV $\rightarrow$ CTV $\rightarrow$ PTV) and deviations in every step can lead to cumulation or a mutual compensation of errors. For example, in one case, the addition of an incorrect GTV, which was too large, and an incorrectly too small CTV margin $(5 \mathrm{~mm})$ resulted in a nearly correct and acceptable CTV/PTV (Supplementary Figure 3).

Taking all these findings into account, it appears necessary to renew the criteria for tumor bed RT-QC. Therefore, we have proposed appropriate definitions helping to better display relevant findings in a structured way in the future (Table 3). According to our proposal for CSI, modification of the PTV should be recommended if the reference CTV is not covered [13]. Moreover, a cutoff for too large PTVs needs to be defined depending on the location of the RT volume and, accordingly, to the expected increase in the risk 
Table 3 Proposal for the definition of acceptable versus unacceptable deviations

Target volume delineation

Step 1

$\begin{array}{lll} & \begin{array}{l}\text { Acceptable deviation } \\ \text { (as a single devia- } \\ \text { tion) }\end{array} & \begin{array}{l}\text { Unacceptable devia- } \\ \text { tion }\end{array} \\ \begin{array}{l}\text { GTV } \\ \text { Getup error margin } \\ \text { not encompass }\end{array} & >\text { setup error margin } \\ \begin{array}{l}\text { MRI visible re- } \\ \text { section cavity/ }\end{array} & & \\ \text { residual tumor } & & >0.5 \mathrm{~cm} \\ \begin{array}{l}\text { GTV tumorbed en- } \\ \text { compasses un- }\end{array} & \leq 0.5 \mathrm{~cm} & \\ \begin{array}{l}\text { involved normal } \\ \text { brain tissue }\end{array} & & \\ \text { CTV margin } & \geq 0.5 \mathrm{~cm}-\leq 1.5 \mathrm{~cm} & <0.5 \mathrm{~cm} \text { or }>1.5 \mathrm{~cm} \\ \text { PTV margin } & \geq 0.2 \mathrm{~cm}-\leq 1 \mathrm{~cm} & <0.2 \mathrm{~cm} \text { or }>1 \mathrm{~cm}\end{array}$

Step $2 \rightarrow$ If more than 1 deviation is present in step 1, it is recommended to create a correct reference CTV/PTV for final solution according to the following criteria

Acceptable deviation

CTV/PTV

Reference CTV not encompassed by CTV but by PTV or

PTV larger than necessary $(\leq 0.5 \mathrm{~cm})$
Unacceptable deviation

Reference CTV not encompassed by PTV

or PTV substantially larger than necessary $(>0.5 \mathrm{~cm})$

Dose uniformity

\begin{tabular}{lll}
$\mathbf{V 9 5} \%$ & $\geq 95$ to $<98 \%$ & $<95 \%$ \\
$\mathbf{V 1 0 7 \%}$ & $>5 \%$ to $<10 \%$ & $\geq 10 \%$ \\
\hline
\end{tabular}

MRI magnetic resonance imaging, GTV gross tumor volume, $C T V$ clinical target volume, PTV planning target volume, V95 volume of PTV receiving $\geq 95 \%$ of the prescribed dose, V107 volume of PTV receiving $\geq 107 \%$ of the prescribed dose

of late toxicity (e.g., $0.5 \mathrm{~cm}$ inside the posterior fossa). In case of two or more deviations (GTV, CTV margin, and/or PTV margin), the potential addition of variations or mutual compensation of errors has to be taken into account, too. Therefore, it is recommended to create a correct reference CTV/PTV for final decision on these cases.

Regarding dose uniformity, we found appropriate dose coverage in most of the cases. Therefore, we propose using stricter dose uniformity constraints (V95\% $\geq 98 \%$ ), as is recommended by the ICRU report 83 for intensity-modulated RT techniques (IMRT) and as proposed for CSI when using high-precision techniques $[13,23]$. The same constraints shall also be used for proton plans, respecting the recommendations of the ICRU 78 report [24].

\section{Impact of experience in treating SIOP PNET5 MB patients}

In contrast to our experiences in RT-QC of CSI plans and the published results for atypical and malignant meningioma, we did not see any difference in the need for plan modifications when comparing low-recruiting and high-recruiting centers ( $\leq 4$ patients $42.3 \%$ vs. $\geq 5$ patients $35.9 \%$ ) $[8,13]$. However, the majority of unacceptable plans $(88 \%)$ occurred in patients who were among the first 3 patients of each institution. However, in 3 cases, plan modification was also recommended at a later stage of study participation. This is in contrast to our experiences with RT-QC in CSI plans, where no unacceptable plan occurred in a fifth or later patient of the respective institution [13]. This might be explained by the highly standardized contouring and planning process for CSI, whereas focal RT of TB is highly individual for the patients. However, the number of reviewed and rejected plans at a later stage of study participation is too low to come to reliable conclusions.

\section{Limitations of the study}

The most important limitation is the reduced clinical significance of the study results due to the rapidly evolving irradiation technique. The first protocol versions included only review criteria of Carrie et al. for simulation-based CSI planning [3]. Dose uniformity criteria of this analysis were established according to the recommendations of the ICRU50/62 for 3D conformal irradiation [14]. However, in most of the cases, high-precision techniques like IMRT or proton beam therapy were used. Therefore, the proposed new constraints are based on the stricter recommendations of the ICRU 83 report [23]. As RT techniques will continue to develop, review criteria will have to be adapted, e.g., robust planning in proton beam therapy.

Another weakness is the incomplete availability of MRI data and the original MRI co-registration matrix, which cause uncertainties in the evaluation of the GTV (tumor bed).

\section{Conclusion}

Comparable to our experiences in RT-QC of CSI plans, our findings on TB boost emphasize the impact of central pretreatment RT-QC in at least the first 3 to 5 patients per institution to ensure protocol-compliant treatment planning for clinical trials in MB [13]. The development of standardized RT-QC criteria, as proposed by us, enables consistent plan review and thereby may be of help for other studies. An MRI at the time of CT-based treatment planning of RT could improve the precision of target volume delineation. 
Supplementary Information The online version of this article (https:// doi.org/10.1007/s00066-021-01822-0) contains supplementary material, which is available to authorized users.

Acknowledgements The authors thank all attending physicians and physicists of the participating centers for their cooperation and the Deutsche Kinderkrebsstiftung (German Childhood Cancer Foundation) for its support.

Funding The analysis was supported by the German Childhood Cancer Foundation (Deutsche Kinderkrebsstiftung).

Funding Open Access funding enabled and organized by Projekt DEAL.

\section{Declarations}

Conflict of interest S. Dietzsch reports grants from the German Childhood Cancer Foundation (Deutsche Kinderkrebsstiftung; DKS2016.01A) during the conduct of the study. A. Braesigk reports grants from the German Childhood Cancer Foundation (Deutsche Kinderkrebsstiftung; DKS2014.18) during the conduct of the study. T. Schlender reports grants from the German Childhood Cancer Foundation (Deutsche Kinderkrebsstiftung; DKS2018.04) during the conduct of the study. M. Mynarek reports grants from the German Childhood Cancer Foundation (Deutsche Kinderkrebsstiftung; DKS2013.15) during the conduct of the study. D. Obrecht reports grants from the German Childhood Cancer Foundation (Deutsche Kinderkrebsstiftung; DKS2013.15) during the conduct of the study. S. Rutkowski reports grants from the German Childhood Cancer Foundation (Deutsche Kinderkrebsstiftung; DKS2013.15) during the conduct of the study. C. Seidel, J. Remmele, R. Kitzing, D. Geismar, K. Jablonska, R. Schwarz, M. Pazos, D. C. Weber, S. Frick, K. Gurtner, C. Matuschek, S. B. Harrabi, A. Glück, V. Lewitzki, K. Dieckmann, M. Benesch, N. U. Gerber, B. Timmermann, and R.-D. Kortmann declare that they have no competing interests.

Ethical standards All procedures performed in studies involving human participants or on human tissue were in accordance with the ethical standards of the institutional and/or national research committee and with the 1975 Helsinki declaration and its later amendments or comparable ethical standards. The SIOP PNET5 MB trial (ClinicalTrials.gov identifier: NCT02066220) was approved by the Ethics committee Hamburg. Informed consent was obtained from all individual participants included in the study.

Open Access This article is licensed under a Creative Commons Attribution 4.0 International License, which permits use, sharing, adaptation, distribution and reproduction in any medium or format, as long as you give appropriate credit to the original author(s) and the source, provide a link to the Creative Commons licence, and indicate if changes were made. The images or other third party material in this article are included in the article's Creative Commons licence, unless indicated otherwise in a credit line to the material. If material is not included in the article's Creative Commons licence and your intended use is not permitted by statutory regulation or exceeds the permitted use, you will need to obtain permission directly from the copyright holder. To view a copy of this licence, visit http://creativecommons.org/licenses/by/4. $0 /$.

\section{References}

1. Northcott PA, Robinson GW, Kratz CP et al (2019) Medulloblastoma. Nat Rev Dis Primers 5(1):11. https://doi.org/10.1038/ s41572-019-0063-6
2. Michalski JM, Janss A, Vezina G et al (2016) Results of COG ACNS0331: a phase III trial of involved-field radiotherapy (IFRT) and low dose craniospinal irradiation (LD-CSI) with chemotherapy in average-risk Medulloblastoma: a report from the children's oncology group. Int J Radiat Oncol Biol Phys 96(5):937-938. https:// doi.org/10.1016/j.ijrobp.2016.09.046

3. Carrie C, Hoffstetter S, Gomez F et al (1999) Impact of targeting deviations on outcome in medulloblastoma: study of the French Society of Pediatric Oncology (SFOP). Int J Radiat Oncol Biol Phys 45(2):435-439

4. Taylor RE, Bailey CC, Robinson KJ et al (2004) Impact of radiotherapy parameters on outcome in the International Society of Paediatric Oncology/United Kingdom Children's Cancer Study Group PNET-3 study of preradiotherapy chemotherapy for M0-M1 medulloblastoma. Int J Radiat Oncol Biol Phys 58(4):1184-1193. https:// doi.org/10.1016/j.ijrobp.2003.08.010

5. Donahue B, Marymont MAH, Kessel S et al (2012) Radiation therapy quality in CCG/POG intergroup 9961: implications for craniospinal irradiation and the posterior fossa boost in future medulloblastoma trials. Front Oncol 2:185. https://doi.org/10.3389/fonc. 2012.00185

6. Carrie C, Muracciole X, Gomez F et al (2005) Conformal radiotherapy, reduced boost volume, hyperfractionated radiotherapy, and online quality control in standard-risk medulloblastoma without chemotherapy: results of the French M-SFOP 98 protocol. Int J Radiat Oncol Biol Phys 63(3):711-716. https://doi.org/10.1016/j. ijrobp.2005.03.031

7. Fairchild A, Weber DC, Bar-Deroma R et al (2012) Quality assurance in the EORTC 22033-26033/CE5 phase III randomized trial for low grade glioma: the digital individual case review. Radiother Oncol 103(3):287-292. https://doi.org/10.1016/j.radonc.2012. 04.002

8. Coskun M, Straube W, Hurkmans CW et al (2013) Quality assurance of radiotherapy in the ongoing EORTC 22042-26042 trial for atypical and malignant meningioma: results from the dummy runs and prospective individual case Reviews. Radiat Oncol 8:23. https://doi.org/10.1186/1748-717X-8-23

9. Gondi V, Cui Y, Mehta MP et al (2015) Real-time pretreatment review limits unacceptable deviations on a cooperative group radiation therapy technique trial: quality assurance results of RTOG 0933. Int J Radiat Oncol Biol Phys 91(3):564-570. https://doi.org/ 10.1016/j.ijrobp.2014.10.054

10. Abrunhosa-Branquinho AN, Bar-Deroma R, Collette S et al (2018) Radiotherapy quality assurance for the RTOG 0834/EORTC 2605322054/NCIC CTG CEC.1/CATNON intergroup trial "concurrent and adjuvant temozolomide chemotherapy in newly diagnosed non1p/19q deleted anaplastic glioma": Individual case review analysis. Radiother Oncol 127(2):292-298. https://doi.org/10.1016/j.radonc. 2018.03.013

11. Paulino AC, Mazloom A, Teh BS et al (2011) Local control after craniospinal irradiation, intensity-modulated radiotherapy boost, and chemotherapy in childhood medulloblastoma. Cancer 117(3):635-641. https://doi.org/10.1002/cncr.25601

12. Merchant TE, Kun LE, Krasin MJ et al (2008) Multi-institution prospective trial of reduced-dose craniospinal irradiation $(23.4 \mathrm{~Gy})$ followed by conformal posterior fossa $(36 \mathrm{~Gy})$ and primary site irradiation $(55.8 \mathrm{~Gy})$ and dose-intensive chemotherapy for averagerisk medulloblastoma. Int J Radiat Oncol Biol Phys 70(3):782-787

13. Dietzsch S, Braesigk A, Seidel C et al (2020) Pretreatment central quality control for craniospinal irradiation in non-metastatic medulloblastoma : first experiences of the German radiotherapy quality control panel in the SIOP PNET5 MB trial. Strahlenther Onkol. https://doi.org/10.1007/s00066-020-01707-8

14. The International Commission of Radiation units and Measurements (1993) ICRU Report 50: Prescribing, Recording and Report- 
ing Photon Beam Therapy. Journal of the ICRU (Volume os-26 Issue 1, September 1993). https://doi.org/10.1093/jicru_os26.1.iii

15. Miralbell R, Bleher A, Huguenin P et al (1997) Pediatric medulloblastoma: radiation treatment technique and patterns of failure. Int J Radiat Oncol Biol Phys 37(3):523-529

16. Miralbell R, Fitzgerald TJ, Laurie F et al (2006) Radiotherapy in pediatric medulloblastoma: quality assessment of Pediatric Oncology Group Trial 9031. Int J Radiat Oncol Biol Phys 64(5):1325-1330. https://doi.org/10.1016/j.ijrobp.2005.11.002

17. Coles CE, Hoole ACF, Harden SV et al (2003) Quantitative assessment of inter-clinician variability of target volume delineation for medulloblastoma: quality assurance for the SIOP PNET 4 trial protocol. Radiother Oncol 69(2):189-194. https://doi.org/10.1016/ j.radonc.2003.09.009

18. Rosenman JG, Miller EP, Tracton G et al (1998) Image registration: an essential part of radiation therapy treatment planning. Int J Radiat Oncol Biol Phys 40(1):197-205. https://doi.org/10.1016/ s0360-3016(97)00546-4

19. Cattaneo GM, Reni M, Rizzo G et al (2005) Target delineation in post-operative radiotherapy of brain gliomas: interobserver variability and impact of image registration of $\mathrm{MR}$ (pre-operative) images on treatment planning CT scans. Radiother Oncol 75(2):217-223. https://doi.org/10.1016/j.radonc.2005.03.012
20. Ulin K, Urie MM, Cherlow JM (2010) Results of a multi-institutional benchmark test for cranial CT/MR image registration. Int J Radiat Oncol Biol Phys 77(5):1584-1589. https://doi.org/10.1016/ j.ijrobp.2009.10.017

21. Scharl S, Kirstein A, Kessel KA et al (2019) Volumenveränderungen der Resektionshöhlen nach Operation von Hirnmetastasen Konsequenzen für die stereotaktische Strahlentherapie (Cavity volume changes after surgery of a brain metastasis-consequences for stereotactic radiation therapy). Strahlenther Onkol 195(3):207-217. https://doi.org/10.1007/s00066-018-1387-y

22. Wee CW, Kim IH, Park C-K et al (2020) Interim tumor progression and volumetric changes of surgical cavities during the surgery-toradiotherapy interval in anaplastic gliomas: implications for additional pre-radiotherapy magnetic resonance imaging. Cancer Res Treat 52(2):524-529. https://doi.org/10.4143/crt.2019.520

23. The International Commission on Radiation Units and Measurements (2010) Prescribing, recording, and reporting intensity-modulated photon-beam therapy (IMRT). J ICRU 10(1):NP. https://doi. org/10.1093/jicru/ndq001

24. International Commission on Radiation Units and Measurements (2007) ICRU report 78: prescribing, recording and reporting proton-beam therapy. J ICRU 7(2):NP. https://doi.org/10.1093/jicru/ ndn001 